\title{
Evidence Suggesting a Role for Hydroxyl Radical in Gentamicin-induced Acute Renal Failure in Rats
}

Patrick D. Walker* and Sudhir V. Shah ${ }^{\ddagger}$

${ }^{*}$ Departments of Pathology and ${ }^{\ddagger}$ Medicine (Nephrology), Tulane University School of Medicine, New Orleans, Louisiana 70112

\begin{abstract}
The protective effect of hydroxyl radical scavengers and iron chelators has strongly implicated the hydroxyl radical in several models of tissue injury. Based on in vitro studies showing gentamicin-enhanced generation of reactive oxygen metabolites in renal cortical mitochondria, we examined the effect of hydroxyl radical scavengers and iron chelators in gentamicininduced acute renal failure. Rats treated with gentamicin (G) alone $(100 \mathrm{mg} / \mathrm{kg}$, s.c. $\times 8 \mathrm{~d})$ developed advanced renal failure (BUN $215 \pm 30 \mathrm{mg} / \mathrm{dl}$ ) compared to saline-treated controls (BUN 16 $\pm 1 \mathrm{mg} / \mathrm{dl}, P<0.001$ ). In contrast, rats treated with gentamicin and either dimethylthiourea (DMTU, an hydroxyl radical scavenger, $125 \mathrm{mg} / \mathrm{kg}$, i.p. twice a day) or deferoxamine (DFO, an iron chelator, $20 \mathrm{mg} /$ day by osmotic pump) had significantly lower BUN (G + DMTU 48.8 $\pm 8 \mathrm{mg} / \mathrm{dl}, P$ $<0.001, n=8$; G + DFO $30 \pm 7 \mathrm{mg} / \mathrm{dl}, P<0.001, n=8$ ). In separate experiments, treatment with two other hydroxyl radical scavengers (dimethyl sulfoxide or sodium benzoate) and a second iron chelator (2,3,dihydroxybenzoic acid) had a similar protective effect on renal function (as measured by both BUN and creatinine). In addition, histological evidence of damage was markedly reduced by the interventional agents. Finally, concurrent treatment with DMTU prevented the gentamicin induced increase in renal cortical malondialdehyde content (G: 4.4 $\pm 0.2 \mathrm{nmol} / \mathrm{mg} ; G+$ DMTU: $3.1 \pm 0.2 \mathrm{nmol} / \mathrm{mg}, P$ $<0.0001, n=8$ ) suggesting that the protective effect of DMTU was related to free radical mechanisms rather than to some other effect. Taken together, these data strongly support a role for hydroxyl radical or a similar oxidant in gentamicininduced acute renal failure.
\end{abstract}

\section{Introduction}

Aminoglycoside antibiotics including gentamicin are widely used in the treatment of gram-negative infections. A major complication of the use of these drugs is nephrotoxicity (reviewed in 1), accounting for $10-15 \%$ of all cases of acute renal failure (1). The specificity of gentamicin for renal toxicity is apparently related to its preferential accumulation in the renal proximal convoluted tubules (50 to 100 times greater than serum) (1). Although gentamicin's effect on biological membranes appears to be critical in the pathogenetic sequence (1),

\section{Address reprint requests to Dr. Walker.}

Presented in part at the Southern Section of the American Federation of Clinical Research, New Orleans, LA, January 1987.

Received for publication 16 March 1987 and in revised form 24 August 1987.

J. Clin. Invest.

(c) The American Society for Clinical Investigation, Inc.

$0021-9738 / 88 / 02 / 0334 / 08 \$ 2.00$

Volume 81, February 1988, 334-341 the exact mechanisms of gentamicin nephrotoxicity are unknown.

A large body of in vitro, ex vivo, and in vivo evidence support the concept that reactive oxygen metabolites including free radical species (e.g., superoxide and hydroxyl radical) and other oxygen metabolites (e.g., hydrogen peroxide, hypochlorous acid) are important mediators of tissue injury (reviewed in 2-4 and 5-13). In particular, recent in vivo studies have demonstrated the protective effect of hydroxyl radical scavengers and/or iron chelators (presumably by preventing the generation of hydroxyl radical by the iron-catalyzed Haber-Weiss reaction) in several models of tissue injury (8-13). These studies thus suggest an important role for hydroxyl radical in tissue injury.

Reactive oxygen metabolites have been shown to affect several biological processes potentially important in glomerular diseases $(14,15)$ and their role in neutrophil-mediated glomerular diseases has recently been demonstrated (16-18). In addition, reactive oxygen metabolites have been postulated to be important in ischemic acute renal failure (19). However, the role of reactive oxygen metabolites in gentamicin-induced nephrotoxicity has not been previously examined.

In our previous studies we have shown that gentamicin in vitro enhances the generation of hydrogen peroxide by renal cortical mitochondria (20). Most, if not all, of the hydrogen peroxide generated by mitochondria is derived from superoxide anion (21). Superoxide anion and hydrogen peroxide may interact (with iron as catalyst, by the Haber-Weiss reaction) to generate the hydroxyl radical $(22,23)$. Several studies have, in fact, shown that agents that enhance the generation of hydrogen peroxide and superoxide anion by mitochondria also enhance the generation of hydroxyl radical $(24,25)$.

Based on the above observations, the aim of the present study was to examine the effect of hydroxyl radical scavengers and iron chelators on gentamicin-induced acute renal failure in rats.

\section{Methods}

Adult male Sprague-Dawley rats weighing 200-250 $\mathrm{g}$ and having free access to water and standard rat chow $(1.00 \%$ calcium, $0.21 \%$ magnesium, $0.40 \%$ sodium, $1.10 \%$ potassium) were used in these experiments. For most studies the rats received daily subcutaneous injections of either $1 \mathrm{ml}$ of sterile, isotonic saline or gentamicin $(100 \mathrm{mg} / \mathrm{kg}$ per d) for 8 consecutive $\mathrm{d}$ (26). $24 \mathrm{~h}$ after the last injection, the rats were sacrificed, plasma obtained for the measurement of blood urea nitrogen $(B U N)^{1}$ and/or creatinine and kidneys obtained for histology and for determination of gentamicin levels.

Interventional therapy. The effect of several hydroxyl radical scavengers and of iron chelators on gentamicin-induced acute renal failure

1. Abbreviations used in this paper: BUN, blood urea nitrogen; DFO, deferoxamine B mesylate; DMTU, dimethylthiourea; MDA, malondialdehyde. 
was examined. Dimethylthiourea (DMTU) was administered in a dose of $500 \mathrm{mg} / \mathrm{kg}$ i.p. just before the first gentamicin injection followed by $125 \mathrm{mg} / \mathrm{kg}$ i.p. twice a day. The other hydroxyl radical scavengers used included dimethyl sulfoxide (DMSO) $4 \mathrm{~g} / \mathrm{kg}$ (27) i.p. twice a day, and sodium benzoate $150 \mathrm{mg} / \mathrm{kg}$ i.p. twice a day. The iron chelator deferoxamine B mesylate (DFO) (Desferal; Ciba-Geigy Corp., Summit, NJ) or 2,3 dihydroxybenzoic acid $(13,28)$ was administered intravenously in a dose of $20 \mathrm{mg} / \mathrm{rat}$ just before the first gentamicin injection. At the same time, deferoxamine or 2,3 dihydroxybenzoic acid was administered via an osmotic pump (type 2ML 2; Alza Corp., Palo Alto, CA) that was implanted subcutaneously. The drug were reconstituted at a concentration of $175 \mathrm{mg} / \mathrm{ml}$, and the pumps (with a 2-ml capacity) delivered $\sim 20 \mathrm{mg}$ of the iron chelator per rat per day at continuous rate of $5 \mu \mathrm{l} / \mathrm{h}$. Previous studies have shown that constant plasma levels of the drug are maintained when the deferoxamine is administered by this route (29).

Urea nitrogen and creatinine were measured in plasma samples using the Beckman BUN Analyzer 2 and the Beckman Creatinine Analyzer (Beckman Instruments, Inc., Fullerton, CA).

Gentamicin assay. One part renal cortical homogenate was diluted with nine parts $0.15 \%$ Triton-X in distilled water. A further dilution was then made to produce a protein concentration of $1 \mathrm{mg} / \mathrm{ml}$ and the concentration of gentamicin was determined using a standard enzymatic radiochemical assay (30). (Assays kindly performed by Michael Wilson and Dr. Robert Daum, Section of Infectious Disease, Department of Pediatrics, Tulane Medical School.)

Briefly, ${ }^{14} \mathrm{C}$-labeled acetyl-coenzyme $\mathrm{A}$ is incubated with sample containing gentamicin and gentamicin acetyl transferase for $10 \mathrm{~min}$ allowing all the gentamicin to go to acetyl-gentamicin. The reaction liquid is then pipetted onto a phosphocellulose disc to which acetylgentamicin binds very tightly. After washing to remove unreacted acetyl-coenzyme $\mathrm{A}$, the bound radioactivity is counted in a liquid scintilation counter. The validity of the assay was assessed by measurements of gentamicin in kidney homogenates from the nongentamicin treated rats and a kidney homogenate from a non-gentamicin treated rat to which gentamicin was added in concentrations of $2,5,10,15$, and 20 $\mu \mathrm{g} / \mathrm{ml}$.

Renal cortical lipid peroxidation. We examined the effect of gentamicin administration and the effect of concurrent DMTU administration on renal cortical malondialdehyde content using the experimental protocol of Ramsammy et al. (31). Male Sprague-Dawley rats (as above) were divided into three groups: the first group received saline only (as control); the second group received gentamicin $(100 \mathrm{mg} / \mathrm{kg}$ s.c.) on days 1-4 and were killed $48 \mathrm{~h}$ after the last injection (31); and the third group received, in addition to gentamicin, DMTU (as described above) until the day of sacrifice. At the time of sacrifice, plasma was obtained for urea nitrogen and creatinine concentration and renal cortex was obtained for malondialdehyde content. Malondialdehyde determinations were carried out on the same day.

Malondialdehyde assay. Lipid peroxidation of renal cortex was assessed by measuring malondialdehyde using the thiobarbituric acid assay as in our previous study (32). Briefly, the renal cortex was separated from the medulla, weighed, minced and homogenized in $0.02 \mathrm{M}$ sodium phosphate buffer, pH 7.4 (1:10 wt/vol) using a Potter-Elvehjem smooth glass homogenizer with a motor driven teflon pestle. $1 \mathrm{ml}$ of $17.5 \% \mathrm{TCA}$ and $1 \mathrm{ml}$ of $0.6 \%$ thiobarbituric acid, $\mathrm{pH} 2$, were added to $1 \mathrm{ml}$ of the homogenate containing $1.0-1.7 \mathrm{mg}$ of renal cortical protein. This mixture was placed in a boiling water bath for $15 \mathrm{~min}$ and then allowed to cool. $1 \mathrm{ml}$ of $70 \%$ TCA was added and the mixture allowed to incubate for $20 \mathrm{~min}$. The sample was then centrifuged for 15 min at 2,000 rpm and the optical density of the supernatant read at 534 nm on a spectrophotometer (DMS 100; Varian Associates, Palo Alto, CA) against a reagent blank. The amount of malondialdehyde, expressed in nanomoles, was calculated using a molar extinction coeffcient of $1.56 \times 10^{5} \mathrm{M}^{-1} \mathrm{~cm}^{-1}$ at $535 \mathrm{~nm}$. Malondialdehyde production is expressed as nanomoles per milligram protein (protein content determined by the method of Lowry [33]).

Kidney histology. The kidneys were sectioned and a portion fixed in either $10 \%$ formalin for light microscopy or $3 \%$ glutaraldehyde for electron microscopy. The tissue for light microscopy was dehydrated and embedded in glycol methacrylate. Sections were cut at $2 \mu \mathrm{m}$ and stained with periodic acid-Schiff reagent. The slides were coded and examined without knowledge of the treatment protocol. Tissues for electron microscopy were post-fixed in $1 \%$ osmium tetroxide for $1 \mathrm{~h}$, dehydrated and embedded in maraglas. Silver sections were obtained, stained with lead citrate and uranyl acetate and examined in an electron microscope (300; Philips Electronic Instruments, Inc., Mahwah, NJ).

A light microscopy semiquantitative analysis of the kidney sections was performed using the technique of Houghton et al. (34). The changes seen were limited to the tubulointerstitial areas and were graded as follows: $0=$ normal; $1=$ areas of focal granulovacuolar epithelial cell degeneration and granular debris in tubular lumens with or without evidence of tubular epithelial cell desquamation in small foci ( $<1 \%$ of total tubule population involved by desquamation); 2 $=$ tubular epithelial necrosis and desquamation easily seen but involving less than half of cortical tubules; $3=$ more than half of proximal tubules showing desquamation and necrosis but uninvolved tubules easily found; 4 = complete or almost complete proximal tubular necrosis. In addition to grading the histological changes, the presence or absence of cytoplasmic PAS-positive bodies (confirmed to be cytosegresomes by electron microscopy) in the proximal tubule epithelial cells was also recorded.

In vitro studies. We examined the effect of DMTU $(10 \mathrm{mM})$ and deferoxamine $(1 \mathrm{mg} / \mathrm{ml})$ on gentamicin-enhanced generation of hydrogen peroxide by renal cortical mitochondria. Mitochondria were isolated as described by Johnson and Lardy (35) using $0.27 \mathrm{M}$ sucrose, $1 \mathrm{mM}$ EGTA, $5 \mathrm{mM}$ Tris- $\mathrm{HCl}$ buffer $\mathrm{pH} 7.4$ as the isolation medium and centrifugation of the $600 \mathrm{~g} \times 10 \mathrm{~min}$ supernatant at $10,000 \mathrm{~g}$ for 10 min to sediment the mitochondria. The mitochondrial pellet was resuspended in $0.25 \mathrm{M}$ sucrose and centrifuged at $10,000 \mathrm{~g}$ for $10 \mathrm{~min}$. The final pellet was suspended in $0.25 \mathrm{M}$ sucrose to give a protein concentration of about $10 \mathrm{mg}$ of protein $/ \mathrm{ml}$. Only mitochondria that had a respiratory control index (state $3 /$ state 4 respiration) of $>2.5$ were used for experiments.

Hydrogen peroxide production was measured using the scopoletin method (36). $0.5 \mathrm{mg}$ of mitochondrial protein was added to the reaction mixture consisting of $150 \mathrm{mM} \mathrm{KCl}, 10 \mathrm{mM}$ Tris-phosphate buffer, $5 \mathrm{mM}$ Tris-HCl buffer, $\mathrm{pH} 7.4$ and $0.1 \mathrm{ml}$ of horseradish peroxidase $(400 \mu \mathrm{g} / \mathrm{ml})$ in a total volume of $3 \mathrm{ml}$. Scopoletin was added $(5$ $\mathrm{nmol}$ ) and the $100 \%$ baseline fluorescence was set in a Spectrofluorometer (Farrand System 3; Farrand Optical Co., Valhalla, NY). The decrease in fluorescence (excitation wavelength $385 \mathrm{~nm}$ and emission wavelength $460 \mathrm{~nm}$ ) was first recorded after the addition of substrate (10 mM sodium succinate, baseline values) and then gentamicin (4 $\mathrm{mM}$ ) was added to the same reaction mixture. The effect of DMTU (10 $\mathrm{mM})$ and deferoxamine $(1 \mathrm{mg} / \mathrm{ml})$ added prior to the addition of gentamicin, on gentamicin-stimulated production of hydrogen peroxide was examined. The amount of hydrogen peroxide produced was determined using a standard curve obtained for each experiment.

Materials. Drugs and reagents were purchased from the following sources: gentamicin, Schering Corp. (Kenilworth, NJ); 2,3 dihydroxybenzoic acid and 1,3-dimethyl-2-thiourea, Aldrich Chemical Co. (Milwaukee, WI); deferoxamine B mesylate (Desferal), Ciba-Geigy Corp.; $\left[{ }^{14} \mathrm{C}\right]$ acetyl Co-A, ICN Radiochemicals Corp. (Irvine, CA); gentamicin-3-acetyl transferase, Pharmacia Fine Chemicals (Piscataway, $\mathrm{NJ}$ ); sodium benzoate, dimethyl sulfoxide and other reagents and buffers, Sigma Chemical Co. (St. Louis, MO).

\section{Results}

In preliminary studies, we examined the time course of the effect of daily subcutaneous injections of gentamicin (100 $\mathrm{mg} / \mathrm{kg}$ ) (26) on renal function (measured by the BUN concentration $24 \mathrm{~h}$ after the last injection). A marked increase in the 
BUN was noted after eight injections in all the gentamicintreated rats. Based on these data, we examined the effect of various interventions on nephrotoxicity after eight injections of gentamicin. In the first experiment we examined the effect of DMTU, a hydroxyl radical scavenger (10) and of the iron chelator deferoxamine on gentamicin-induced acute renal failure. Rats treated with gentamicin for $8 \mathrm{~d}$ had a marked increase in BUN $(215 \pm 30 \mathrm{mg} / \mathrm{dl}, n=8)$ compared to salinetreated controls (BUN: $16 \pm 1 \mathrm{mg} / \mathrm{dl}, n=8$ ) (Fig. 1). In contrast, rats treated with gentamicin and DMTU (in doses that have previously been shown to achieve concentrations sufficient to scavenge hydroxyl radical in vivo (10)) had significantly lower BUN values (BUN: $48 \pm 17 \mathrm{mg} / \mathrm{dl}, n=8, P$ $<0.0001)$. Similarly, deferoxamine afforded a marked protective effect (BUN: $30 \pm 7 \mathrm{mg} / \mathrm{dl}, n=8, P<0.0001$ ) against gentamicin-induced acute renal failure (Fig. 1).

We considered the possibility that the interventional agents might have caused the gentamicin nephrotoxicity to be manifested at an earlier time point and that the BUN was lower after eight injections because these rats were in the recovery phase. We therefore examined the effect of deferoxamine on rats receiving six gentamicin injections. In the deferoxaminetreated animals, the mean BUN was significantly lower $(22 \pm 2$, $n=6, P<0.0001)$ compared to the mean BUN in rats treated gentamicin alone $(80 \pm 5, n=6)$. In separate experiments we also examined whether the protective effects were present at longer time points. Rats were treated with either gentamicin alone or gentamicin with deferoxamine. By day 11 half of the rats $(6 / 16$ by day 10 and $8 / 16$ by day 11$)$ treated with gentamicin alone had died (presumably from acute renal failure). In contrast, there was no mortality in gentamicin treated rats receiving deferoxamine. When the study was terminated, the BUN in deferoxamine-treated rats was significantly lower (days 10 and $11,48 \pm 3, n=16, P<0.0001$ ) than the BUN from the surviving rats $(50 \%$ mortality) receiving gentamicin alone (days 10 and $11,99 \pm 7, n=8$ ). These results thus provide further evidence for the protective effect of deferoxamine in both mortality and renal failure induced by gentamicin.

We also considered the possibility that the protective effect of DMTU and deferoxamine might be related to an effect on gentamicin uptake by the renal tissue. We therefore measured gentamicin levels in the kidney cortices. The renal cortical gentamicin concentrations from the first study were as follows: gentamicin alone, $14 \pm 0.7 \mu \mathrm{g} / \mathrm{mg}$ protein, $n=8$; gentamicin plus DMTU, $19 \pm 1.9 \mu \mathrm{g} / \mathrm{mg}$ protein, $n=8$; and gentamicin plus deferoxamine, $20 \pm 1.5 \mu \mathrm{g} / \mathrm{mg}$ protein, $n=8$. These results indicate that the protective effect of these agents was unrelated to the uptake of gentamicin by renal cortical tissue. We also

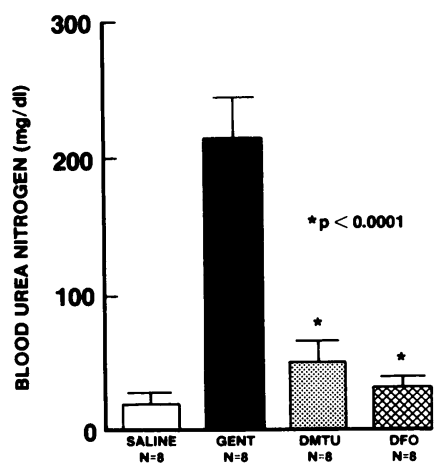

Figure 1.The effect of the hydroxyl radical scavenger DMTU and the iron chelator DFO on gentamicin (GENT)induced acute renal failure as measured by blood urea nitrogen. $N$, number of animals in each group. considered the possibility that the effect of DMTU and deferoxamine may be related to some direct interference with the ability of the mitochondria to respond to gentamicin. We therefore examined in vitro the effect of DMTU and deferoxamine on gentamicin-enhanced generation of hydrogen peroxide by renal cortical mitochondria. DMTU $(10 \mathrm{mM})$ and deferoxamine $(1 \mathrm{mg} / \mathrm{ml})$ had no significant effect on gentamicin-enhanced generation of hydrogen peroxide (data not shown). DMTU $(6,10)$ and deferoxamine $(6)$ have been similarly shown not to affect generation of superoxide and/or hydrogen peroxide by neutrophils. Taken together these results demonstrate a significant protective effect of DMTU and deferoxamine on gentamicin-induced acute renal failure and that this protection is not due to their effect either on the mitochondrial response to gentamicin or on the uptake of gentamicin by renal cortical tissue.

In a second experiment, we examined the effect of DMTU again and a second hydroxyl radical scavenger, DMSO (37), on gentamicin-induced acute renal failure. DMTU provided a marked protective effect (confirming the results described above) with both BUN and plasma creatinine being significantly lower compared to rats treated with gentamicin alone (Fig. 2). Also, in rats concurrently treated with DMSO (which has been previously shown to protect against tissue injury $[8,9$, 11-13]), both BUN and plasma creatinine were significantly lower compared to rats treated with gentamicin alone (Fig. 2).

In a third experiment, we examined the effect of a third hydroxyl radical scavenger, sodium benzoate (13). In addition, we also examined the effect of the iron chelator deferoxamine
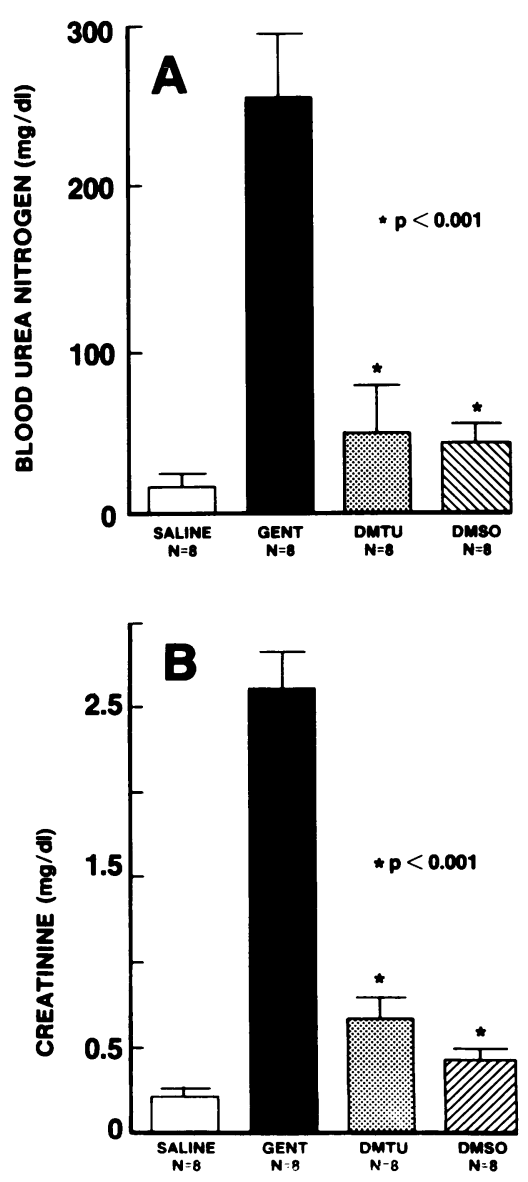

Figure 2. The effect of the hydroxyl radical scavengers DMTU and DMSO on gentamicin (GENT)-induced acute renal failure as measured by $(A)$ BUN and $(B)$ creatinine. $N$, number of animals in each group. 
on gentamicin-induced acute renal failure. The BUN and plasma creatinine were significantly lower in rats that received sodium benzoate or deferoxamine in addition to gentamicin (Fig. 3). In the same experiment, iron-saturated deferoxamine was only partially protective (BUN $64 \pm 6, n=6$ ). In addition, we also examined the effect of a second iron chelator on gentamicin-induced acute renal failure. In rats concurrently treated with 2,3,dihydroxybenzoic acid both BUN $(29 \pm 1.5, n$ $=4)$ and plasma creatinine $(0.6 \pm 0.1, n=4)$ were significantly lower $(P<0.0001)$ compared to rats treated with gentamicin alone (BUN: 205 \pm 9 ; plasma creatinine: $2.6 \pm 0.1, n=4$ ).

In addition to measuring renal function, we also examined the histological changes in the different groups in kidney tissue obtained at the end of the second and the third experiments. The histological changes were graded as described in Methods and the results are expressed in Table I. In rats treated with gentamicin alone for $8 \mathrm{~d}$, the extent of tubular necrosis varied from $<10 \%$ (grade 2) to $>75 \%$ (grades 3 and 4 ) of tubules as described in previous studies (34). In addition, numerous PAS-positive cytoplasmic bodies (cytosegresomes by electron microscopy) were seen in all of the animals in keeping with previous studies that have shown the development of cytosegresomes within $48 \mathrm{~h}$ following the first gentamicin injection (1). In rats treated with the hydroxyl radical scavengers or with deferoxamine (in addition to receiving gentamicin), the PASpositive cytoplasmic bodies were also seen in all the animals; however, as shown in Table I and Fig. 4, there was a marked reduction in the extent of tubular damage.
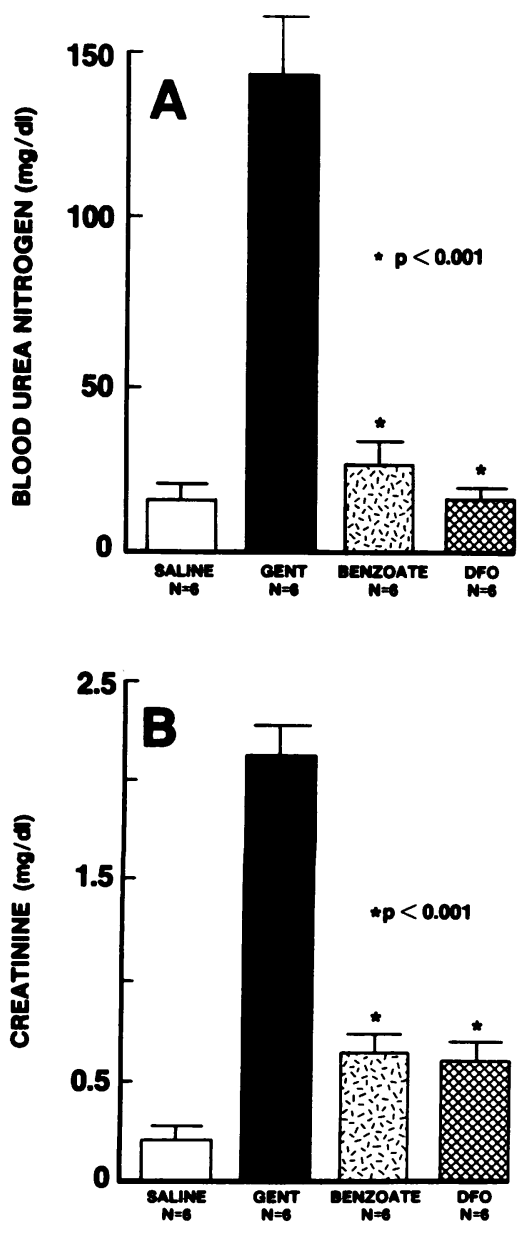

Figure 3. The effect of the hydroxyl radical scavenger sodium benzoate (BENZOATE) and the iron chelator DFO on gentamicin (GENT)-induced acute renal failure as measured by $(A)$ blood urea nitrogen and $(B)$ creatinine. $N$, number of animals in each group.
Table I. Semiquantitative Analysis of Renal Histology*

\begin{tabular}{|c|c|c|c|c|c|}
\hline \multirow[b]{2}{*}{ Group } & \multicolumn{5}{|c|}{ Grade } \\
\hline & 0 & $1+$ & $2+$ & $3+$ & $4+$ \\
\hline Controls & 14 & - & - & - & 一 \\
\hline Gentamicin & - & - & 4 & 4 & 6 \\
\hline Gentamicin + DMTU & 2 & 1 & 4 & 1 & - \\
\hline Gentamicin + DMSO & 8 & - & - & - & - \\
\hline Gentamicin + Benzoate & 4 & - & 1 & 1 & - \\
\hline Gentamicin + DFO & 6 & - & - & - & - \\
\hline
\end{tabular}

* Histologic grading as follows: $0=$ normal; $1=$ Areas of focal granulovacuolar epithelial cell degeneration with $<1 \%$ of total tubule population showing epithelial cell desquamation; 2 = Tubular epithelial necrosis and desquamation involving $<50 \%$ of cortical tubules; 3 = Greater than $50 \%$ of proximal tubules showing desquamation (uninvolved tubules easily found); 4 = Complete or almost complete proximal tubular necrosis. Numerous cytoplasmic PAS-positive bodies were present in the proximal tubule epithelial cells of all gentamicin-treated animals with or without interventional treatment.

Previous studies have demonstrated that there is an increase in renal cortical lipid peroxidation in gentamicin treated rats, and that lipid peroxidation serves as a marker of enhanced free radical generation in response to gentamicin (31). In order to provide additional support for the notion that the protective effect of the hydroxyl radical scavengers was due to their ability to scavenge free radicals rather than some other mechanism, we examined the effect of gentamicin administration, and concurrent DMTU administration on renal cortical lipid peroxidation. Using a time course that has been previously used to demonstrate enhanced lipid peroxidation (31), we found that rats treated with gentamicin alone had a signifcant increase in malondialdehyde content (as measured by the thiobarbituric acid method) compared to saline treated controls (Table II). In rats treated concurrently with DMTU, the malondialdehyde content was significantly lower compared to gentamicin treated rats and similar to the malondialdehyde content in saline-treated rats. In the same experiment, DMTU was protective against gentamicin-induced renal failure as measured by BUN and plasma creatinine (Table II). In a separate in vitro experiment, we confirmed that DMTU did not interfere with malondialdehyde determination (data not shown).

\section{Discussion}

We examined the effect of hydroxyl radical scavengers and iron chelators on gentamicin-induced acute renal failure. In two separate studies, DMTU and deferoxamine provided marked functional and histological protection against acute renal failure in rats receiving gentamicin for $8 \mathrm{~d}$. The protective effect of DMTU and deferoxamine was not due to their effect either on the mitochondrial response to gentamicin or on the uptake of gentamicin by renal cortical tissue. Two additional hydroxyl radical scavengers, sodium benzoate and DMSO as well as a second iron chelator, 2,3 dihydroxybenzoic acid, also afforded a marked protection against gentamicin-induced acute renal failure. Finally the protective effect of deferoxamine was also noted at earlier (6 d) and at later (10 and 11 d) time points. 

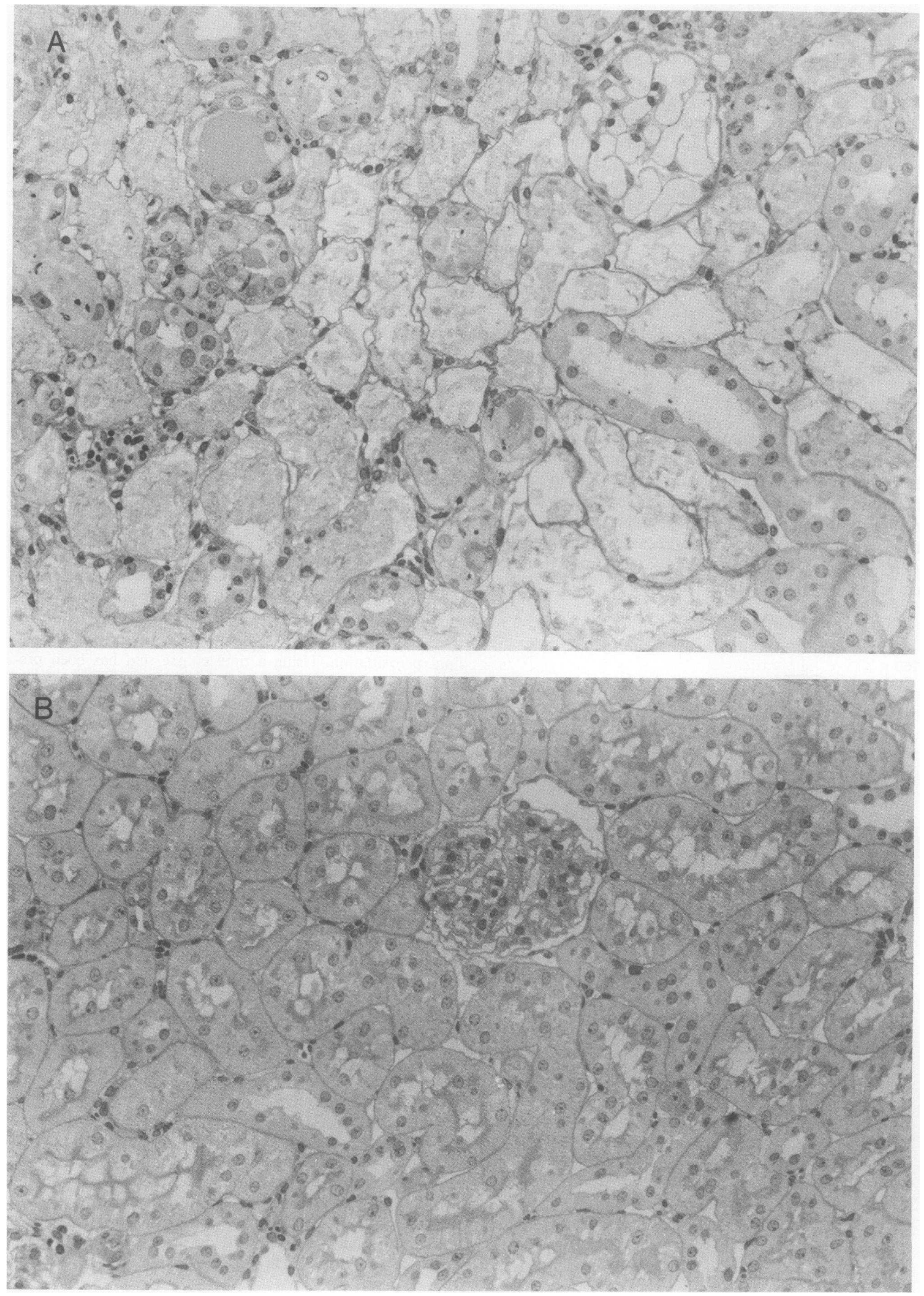
Table II. Renal Cortical MDA Content in Rats Treated with Either Gentamicin Alone or Concurrently with DMTU*

\begin{tabular}{lllll}
\hline & $n$ & MDA & BUN & Creatinine \\
\hline & & $n m o l / m g$ & $m g / d l$ & $m g / d l$ \\
Saline control & 8 & $2.7 \pm 0.1$ & $13 \pm 1$ & $0.2 \pm 0.02$ \\
Gentamicin & 8 & $4.4 \pm 0.2^{\ddagger}$ & $77 \pm 5^{\ddagger}$ & $1.4 \pm 0.07^{\ddagger}$ \\
Gentamicin + DMTU & 8 & $3.1 \pm 0.2^{\S}$ & $16 \pm 1^{\S}$ & $0.3 \pm 0.03^{\S}$
\end{tabular}

* Gentamicin $(100 \mathrm{mg} / \mathrm{kg}$, s.c. $) \times 4$ d. Sacrificed $48 \mathrm{~h}$ after last gentamicin injection. DMTU administered until the day of sacrifice. All values are mean \pm standard error of the mean.

${ }^{\ddagger}$ Gentamicin vs. saline control, $P<0.0001$ (unpaired Student's $t$ test).

${ }^{\S}$ Gentamicin vs. gentamicin + DMTU, $P<0.0001$ (unpaired Student's $t$ test).

Both DMTU, a potent hydroxyl radical scavenger, and the iron chelator deferoxamine, have been extensively used in recent studies to delineate a potential role for hydroxyl radical in several models of tissue injury $(5,8-13)$. The ability of DMTU to scavenge hydroxyl radical has been well established in in vitro studies (10). In addition, DMTU has been shown to achieve concentrations sufficient to scavenge hydroxyl radical in vivo (half life-34 hours in rats) (10). DMTU has been successfully used to prevent acute lung injury in rats after exposure to a variety of injurious agents including: hyperoxia (10), thermal trauma (13), or, in isolated rat lung preparations, enzymatically generated oxygen metabolites (5) or stimulated neutrophils (10).

Similarly, iron chelators, including deferoxamine and 2,3 dihydroxybenzoic acid, have been shown to be protective in several in vivo models of tissue injury $(8,9,11-13)$. The protective effect of iron chelators has been generally taken as evidence for the participation of hydroxyl radical in tissue injury because iron is critical in the generation of hydroxyl radical (via the Haber-Weiss reaction). Indeed, in in vitro studies, the ability of deferoxamine to block the generation of hydroxyl radical has been well demonstrated (22). Although iron-mediated tissue injury could also be explained by a reaction involving the formation of the perferryl ion (23), in our study, the effectiveness of several hydroxyl radical scavengers suggests that the role of iron is related to its participation in the generation of hydroxyl radical. Taken together, the marked protective effect of both the hydroxyl radical scavengers and the iron chelators strongly implicate a role for hydroxyl radical in gentamicin-induced acute renal failure.

An important question related to our results is the source of the reactive oxygen metabolites. In our previous studies we have shown that gentamicin in vitro enhances the generation of hydrogen peroxide by renal cortical mitochondria (20). Although we have not measured the generation of hydroxyl radical by renal cortical mitochondria in response to gentamicin, several studies have, in fact, shown that agents that enhance the generation of hydrogen peroxide and superoxide anion by mitochondria also enhance the generation of hydroxyl radical $(24,25)$. Thus a likely source for the hydroxyl radical appears to be the renal cortical mitochondria. However, other sources for the hydroxyl radical cannot be excluded. One possibility is that the enhanced generation of hydroxyl radical is by leukocytes since the role of leukocytes in gentamicin-induced acute renal failure has never been critically examined.

Although the marked protective effects of iron chelators in several models of tissue injury imply an important role for iron, both the source of physiological iron in vivo, and how it becomes biologically available to participate in the generation of hydroxyl radical remain enigmatic. Recent studies have begun to examine these important issues. Gutteridge et al. (38) have developed an assay, based on the use of the antibiotic bleomycin, to detect iron complexes capable of catalyzing radical reactions in biological fluids. Using this assay they have demonstrated that nonprotein bound iron, which has the potential to support hydroxyl radical generation, may be detected in cerebrospinal, synovial, and inflammatory fluids. In addition, ferritin, generally regarded as a secure means of storing iron in an inert form, may play a more dynamic role in vivo. For example, mitochondria contain distinct binding sites for ferritin from which iron can be mobilized $(39,40)$. Recent studies also indicate that reactive oxygen metabolites may play a role in the release of iron from ferritin. For example, superoxide, generated by xanthine-xanthine oxidase, was shown to release ferritin iron in vitro $(41,42)$ and the released iron $\left(\mathrm{Fe}^{2}\right)$ reacted with hydrogen peroxide to form hydroxyl radical (23). In our recent in vitro study we reported that gentamicin markedly enhances the generation of reactive oxygen metabolites by renal cortical mitochondria (20). This suggests the possibility that the reactive oxygen metabolites generated by gentamicin could mobilize iron for participation in the generation of hydroxyl radical. An additional possible source of iron for the generation of hydroxyl radical that must be considered is enhanced uptake of iron by tissue. Indeed, altered trace metal metabolism with increased concentrations of iron in kidney have been reported in other disease states (43). At present the precise source of iron and the how it gets mobilized to participate in the generation of hydroxyl radical in gentamicin-induced acute renal failure remains unknown.

We did not examine the biological processes that may be affected by the hydroxyl radical leading to acute renal failure. One of the mechanisms by which hydroxyl radical has been postulated to cause tissue damage is by causing peroxidation of membrane lipids. Although lipid peroxides are potentially harmful to tissues and cells and they have been found to be increased in several models of tissue injury $(8,13)$, it has not always been possible to implicate them directly as the cause of tissue injury. For example, Till et al. who examined lipid peroxidation and acute lung injury concluded that lipid peroxidation appeared to be a marker of oxygen-mediated tissue injury rather than to mediate or propagate the tissue injury (13). Similarly in gentamicin-treated rats, there is an increase in the malondialdehyde content (TBA-reactive material) in the kidney cortex. Ramsammy and colleagues have examined the

Figure 4. (A) Light microscopy section of kidneys from rats receiving gentamicin alone showing severe tubular epithelial necrosis with sloughing of the lining epithelium and luminal debris (grade 4, periodic acid-Schiff stain $\times 200$ ). (B) Light microscopy section of kidneys from rats receiving gentamicin plus deferoxamine showing essentially no pathologic abnormalities (grade 0 , periodic acid-Schiff stain $\times 200$ ). 
role of lipid peroxidation in gentamicin induced acute renal failure by examining the effect of diphenyl-phenylenediamine (31) and vitamin E (44), which are potent lipid antioxidants and protect against lipid peroxidation (43). In these studies, both diphenyl-phenylenediamine (31) and vitamin E (44) prevented gentamicin-induced lipid peroxidation, but did not prevent the acute renal failure. Although these studies indicate that lipid peroxidation is unlikely to be the mechanism of gentamicin-induced injury, lipid peroxidation nonetheless serves as marker of enhanced free radical production by renal cortical tissue in response to gentamicin. Our data confirm that the malondialdehyde content is increased in gentamicintreated animals; furthermore, the protective effect of the hydroxyl radical scavenger DMTU was associated with significant reduction in the malondialdehyde content in the renal cortex. These results provide support for the notion that the protective effect of DMTU was related its ability to scavenge free radicals rather than to some other mechanism.

It should be noted that lipid peroxidation is not the exclusive mechanism for oxygen radical-induced damage to the tissues. In particular, hydroxyl radical is one of the most reactive chemical species known and is capable of reacting at extremely high rate-constants with almost every type of molecule found in living cells (e.g., sugars, amino acids, phospholipids, nucleotides, organic acids, etc.) $(46,47)$. It can thus potentially affect numerous cellular processes leading to altered cellular function and/or cell death. Our study, which shows the protective effects of several hydroxyl radical scavengers and iron chelators coupled with studies by other investigators showing the lack of a protective effect of vitamin $E$ and diphenyl-phenylenediamine $(31,44)$, suggest that the role of hydroxyl radical in gentamicin-induced acute renal failure is most likely due to its effects on biological processes other than lipid peroxidation.

\section{Acknowledgments}

We thank Clare Das for technical assistance and Michael Wilson and Dr. Robert Daum for performance of the gentamicin assays.

Supported in part by the Louisiana Heart Association. Dr. Shah is the recipient of the Established Investigator Award from the American Heart Association with funds contributed in part by the Louisiana Heart Association.

\section{References}

1. Humes, H. D., and J. M. Weinberg. 1986. Toxic Nephropathies. In The Kidney. B. M. Brenner and F. C. Rector, Jr., editors. W. B. Saunders Co., Philadelphia. 1491-1532.

2. Fantone, J. C., and P. A. Ward. 1982. Role of oxygen-derived free radicals and metabolites in leukocyte-dependent inflammatory reactions. Am. J. Pathol. 107:397-418.

3. Fantone, J. C., and P. A. Ward. 1985. Polymorphonuclear leukocyte-mediated cell and tissue injury. Oxygen metabolites and their relations to human disease. Hum. Pathol. 16:973-978.

4. Weiss, S. J., and A. F. LoBuglio. 1982. Phagocyte-generated oxygen metabolites and cellular injury. Lab. Invest. 47:5-18.

5. Tate, R. M., K. M. Vanbenthuysen, D. M. Shasby, I. F. McMurtry, and J. G. Repine. 1982. Oxygen-radical-mediated permeability edema and vasoconstriction in isolated perfused rabbit lungs. Am. Rev. Respir. Dis. 126:802-806.

6. Varani, J., S. E. G. Fligiel, G. O. Till, R. G. Kunkel, U. S. Ryan, and P. A. Ward. 1985. Pulmonary endothelial cell killing by human neutrophils. Possible involvement of hydroxyl radical. Lab. Invest. 53:656-663.
7. Martin, W. J. 1983. Nitrofurantoin: Evidence for the Oxidant Injury of Lung Parenchymal Cells. Am. Rev. Respir. Dis. 127:482-486.

8. Ward, P. A., G. O. Till, J. R. Hatherill, T. M. Annesley, and R. G. Kunkel. 1985. Systemic complement activation, lung injury, and products of lipid peroxidation. J. Clin. Invest. 76:517-527.

9. Ward, P. A., G. O. Till, R. Kunkel, and C. Beauchamp. 1983. Evidence for role of hydroxyl radical in complement and neutrophildependent tissue injury. J. Clin. Invest. 72:789-801.

10. Fox, R. B. 1984. Prevention of granulocyte-mediated oxidant lung injury in rats by a hydroxyl radical scavenger, dimethylthiourea. J. Clin. Invest. 74:1456-1464.

11. Fligiel, S. E. Q., P. A. Ward, K. J. Johnson, and G. O. Till. 1984. Evidence for a role of hydroxyl radical in immune-complex-induced vasculitis. Am. J. Physiol. 115:375-382.

12. Johnson, K. J., P. A. Ward, R. G. Kunkel, and B. S. Wilson. 1986. Mediation of IgA induced lung injury in the rat. Role of macrophages and reactive oxygen products. Lab. Invest. 54:499-506.

13. Till, G. O., J. R. Hatherill, W. W. Tourtellotte, M. J. Lutz, and P. A. Ward. 1985. Lipid peroxidation and acute lung injury after thermal trauma to skin. Am. J. Physiol. 119:376-384.

14. Shah, S. V. 1984. Effect of enzymatically generated reactive oxygen metabolites on the cyclic nucleotide content in isolated rat glomeruli. J. Clin. Invest. 74:393-401.

15. Shah, S. V., W. H. Baricos, and A. Basci. 1987. Degradation of human glomerular basement membrane by stimulated neutrophils: Activation of a metalloproteinase/s by reactive oxygen metabolites. $J$. Clin. Invest. 79:25-31.

16. Rehan, A., K. J. Johnson, R. C. Wiggins, R. G. Kunkel, and P. A. Ward. 1984. Evidence for the role of oxygen radicals in acute nephrotoxic nephritis. Lab. Invest. 51:396-403.

17. Rehan, A., K. J. Johnson, R. G. Kunkel, and R. C. Wiggins. 1985. Role of oxygen radicals in phorbol myristate acetate-induced glomerular injury. Kidney Int. 27:503-511.

18. Rehan, A., R. C. Wiggins, R. G. Kunkel, G. O. Till, and K. J. Johnson. 1986. Glomerular injury and proteinuria in rats after intrarenal injection of cobra venom factor. Am. J. Physiol. 123:57-66.

19. Paller, M. S., J. R. Hoidal, and T. F. Ferris. 1984. Oxygen free radicals in ischemic acute renal failure in the rat. J. Clin. Invest. 74:1156-1164.

20. Walker, P. D., and S. V. Shah. 1987. Gentamicin enhanced production of hydrogen peroxide by renal cortical mitochondria. Am. J. Physiol. 253:495-499.

21. Forman, H. J., and A. Boveris. 1982. Superoxide radical and hydrogen peroxide in mitochondria. In Free Radicals in Biology. Academic Press, Inc. New York. 65-90.

22. Hoe, S., D. A. Rowley, and B. Halliwell. 1982. Reactions of ferrioxamine and desferrioxamine with the hydroxyl radical. Chem. Biol. Interactions. 41:75-81.

23. Aust, S. D., L. A. Morehouse, and C. E. Thomas. 1985. Role of metals in oxygen radical reactions. J. Free Radicals Biol. Med. 1:3-25.

24. Doroshow, J. H., and K. J. A. Davies. 1986. Redox cycling of anthracyclines by cardiac mitochondria. J. Biol. Chem. 261:30683074.

25. Komiyama, T., T. Kikuchi, and Y. Sugiura. 1982. Generation of hydroxyl radical by anticancer quinone drugs, carbazilquinone, mitomycin C, aclacinomycin A and adriamycin, in the presence of NADPH-cytochrome P-450 reductase. Biochem. Pharmacol. 31:3651-3656.

26. Humes, H. D., M. Sastrasinh, and J. M. Weinberg. 1984. Calcium is a competitive inhibitor of gentamicin-renal membrane binding interactions and dietary calcium supplementation protects against gentamicin nephrotoxicity. J. Clin. Invest. 73:134-147.

27. Lotan, D., B. S. Kaplan, J. S. C. Fong, P. R. Goodyear, and J. P. de Chadarevian. 1984. Reduction of protein excretion by dimethyl sulfoxide in rats with passive Heymann nephritis. Kidney Int. 25:778788.

28. Graziano, J. H., R. W. Grady, and A. Cerami. 1974. The 
identification of 2,3-dihydroxybenzoic acid as a potentially useful iron-chelating drug. J. Pharmacol. Exp. Ther. 190:570-575.

29. Bowern, N., I. A. Ramshaw, I. A. Clark, and P. C. Doherty. 1984. Inhibition of autoimmune neuropathological process by treatment with an iron-chelating agent. J. Exp. Med. 160:1532-1543.

30. Smith, D. H., B. Van Otto, and A. L. Smith. 1972. A rapid chemical assay for gentamicin. N. Engl. J. Med. 286:583-586.

31. Ramsammy, L. S., C. Josepovitz, K. Y. Ling, B. P. Lane, and G. J. Kaloyanides. 1986. Effects of diphenyl-phenylenediamine on gentamicin-induced lipid peroxidation and toxicity in rat renal cortex. J. Pharmacol. Exp. Ther. 238:83-88.

32. Shah, S. V., F. C. Cruz, and W. H. Baricos. 1983. NADPH-induced chemiluminescence and lipid peroxidation in kidney microsomes. Kidney Int. 23:691-698.

33. Lowry, O. H., N. J. Rosebrough, A. L. Farr, R. J. Randall. 1951. Protein measurement with the Folin phenol reagen. J. Biol. Chem. 193:265-275.

34. Houghton, D. C., C. E. Plamp, J. M. DeFehr, W. M. Bennett, G. Porter, and D. Gilbert. 1978. Gentamicin and tobramycin nephrotoxicity. A morphologic and functional comparison in the rat. Am.J. Pathol. 93:137-152.

35. Johnson, D., and H. Lardy. 1967. Isolation of liver or kidney mitochondria. Methods Enzymol. 10:94-96.

36. Boveris, A., E. Martino, and O. M. Stoppani. 1973. Evaluation of the horseradish peroxidase-scopoletin method for the measurement of hydrogen peroxide formation in biological systems. Anal. Biochem. 80:145-158.

37. Repine, J. E., J. W. Eaton, M. W. Andes, J. R. Hoidal, and R. B. Fox. 1979. Generation of hydroxyl radical by enzymes, chemicals and human phagocytes in vitro. Detection with the anti-inflammatory agent dimethyl sulfoxide. J. Clin. Invest. 64:1642-1651.
38. Gutteridge, J. M. C., D. A. Rowley, and B. Halliwell. 1982. Superoxide-dependent formation of hydroxyl radicals and lipid peroxidation in the presence of iron salts. Biochem. J. 206:605-609.

39. Ulvik, R. J., and I. Romslo. 1979. Studies on the mobilization of iron from ferritin by isolated rat liver mitochondria. Biochim. Biophys. Acta. 588:256-271.

40. Ulvik, R. J. 1982. Relevance of ferritin-binding sites on isolated mitochondria to the mobilization of iron from ferritin. Biochim. Biophys. Acta. 715:42-51.

41. Biemond, P., A. J. G. Swaak, C. M. Beindorff, and J. F. Koster. 1986. Superoxide-dependent and -independent mechanisms of iron mobilization from ferritin by xanthine oxidase. Biochem. J. 239:169173.

42. Thomas, C. E., L. A. Morehouse, and S. D. Aust. 1985. Ferritin and superoxide-dependent lipid peroxidation. J. Biol. Chem. 260:3275-3280.

43. Johnson, W. T., and G. W. Evans. 1984. Effects of the interrelationship between dietary protein and minerals on tissue content of trace metals in streptozotocin-diabetic rats. J. Nutr. 114:189-190.

44. Ramsammy, L. S., C. Josepovitz, K.-Y. Ling, B. P. Lane, and G. J. Kaloyanides. 1987. Failure of inhibition of lipid peroxidation by vitamin $\mathrm{E}$ to protect against gentamicin nephrotoxicity in the rat. Biochem. Pharmacol. 36:2125-2132.

45. Halliwell, B., and J. M. C. Gutteridge. 1985. Chapter 4. Lipid peroxidation: a radical chain reaction. In Free Radicals in Biology and Medicine. Clarendon Press, Oxford. 139-187.

46. Halliwell, B., and J. M. C. Gutteridge. 1985. The chemistry of oxygen radicals and other oxygen-derived species. In Free Radicals in Biology and Medicine. Clarendon Press, Oxford. 20-64.

47. Pryor, W. A. 1986. Oxy-radicals and related species: their formation, lifetimes, and reactions. Annu. Rev. Physiol. 48:657-667. 